\title{
Plant species distribution and their relation to soil properties in Wadi Elip South Western area in Saudi Arabia
}

\author{
Ali A. Al- Masaudi, H.S.Al-Zahrani, A.A. Al Toukhy and Hameed Alsamadany \\ Department of Biological Sciences, Faculty of Science, King Abdulaziz University, Jeddah,SaudiArabia
}

Correspondence Author: Abdul Moneam A. Al-Toukhy, Department of Biological Sciences, Faculty of Science, King Abdulaziz University, Jeddah,SaudiArabia

E-mail: abdulmonemtoukhy@yahoo.com

Received date: 23 January 2018, Accepted date: 27 March 2018, Online date: 10 April 2018

Copyright: (C) 2018 A. Ali A. Al- Masaudi et al. This is an open-access article distributed under the terms of the Creative Commons Attribution License, which permits unrestricted use, distribution, and reproduction in any medium, provided the original author and source are credited.

\begin{abstract}
Soil characteric and flora in Wadi Elip that located in South Western of Saudi Arabiawas investigatedto study the relationships between soil properties and plant types. Results showed that three soil units in study area, foothill, coastal sand dunes and coastal sabkha were found. Soil texture in foothills \& coastal sand dunes was sandy \& sand loamy but in coastal sabkha it was loamy, Bulk density values ranged from $1.12-1.07,1.10-1.07-1.40-1.35 \mathrm{~g} / \mathrm{cm}^{3}$, whereas real density varied between $2.88-2.28,2.88-2.33$ and $2.45-2.40 \mathrm{~g} / \mathrm{cm}^{3}$. Total porosity values ranging from $60.00-53.07,62.85-52.79$ and $43.75-42.86 \%$,pH Values ware $6.7,7.20$ and 8.40 for three soil units mentioned respectively.These results indicating that soils were moderately to strongly alkaline. Ec values ranges from 0.10 to $8.20 \mathrm{Desm}$. $/ \mathrm{m}$. indicated that soils were normal to high sainality, organic carbone was highly in Coastal sabkha near Red Sea (6.30\%) and slightly highly in coastal sand dunes (3.8\%) and it was low (0.90\%) in sand plains, sodium was very highly concentration near The Red Sea (9.335 ml/g), also Mg,Ca,K,p ware highly near TheRed Sea ( 1.372,1.49,0.975 and 3.040) respectively.Aervajavanica, and Prosopis juliflora in foothills area,Salvadorapersica found coastal sand dunes (meidle study area), Suaedafruticosa in coastal sabkha (near The Red Sea) were found. Soil types at the study area were determined to be sandy immature soil. Their horizon sequences were described as surface, subsurface and bottom layers, Coastal sabkha and coastal sand dunes were characterized by a relatively high calcium concentration in the soil horizons. The subsurface layers in coastal sabkha and coastal sand dunes were characterized by founding slightly highly organic carbon accumulation. The roots in the coastal sabkha and coastal sand dunes were distributed more shallowly than those in the sand plains and wadi areas, thus avoiding the high water table level as well as the influence of seawater in the soil. Generally, the salt marsh showed high sodium(9.335 mg/g) concentration and base saturation, indicating that this area was directly affected by seawater.
\end{abstract}

Key words: Soil texture.WadiElip.wild plants.salt effect. organic matter

\section{INTRODUCTION}

Several distinct physiographical regions, such as mountains, valleys (wadis), sandy and rocky deserts, salt (Sabkhas), lava areas (Harrats) covers Saudi Arabia. It is a large dry desert with total area of about 2.25 million $\mathrm{km}^{2}$ covering the major part of the Arabian Peninsula [1,2]. SaudiArabia Lat. 32' $34^{\circ} \mathrm{N}-16^{\prime}$ $83^{\circ} \mathrm{N}$, Long. $34^{\prime} 36^{\circ} \mathrm{E}-56^{\prime} \mathrm{E}$, it is lie in the dry and semi dry part of the world, significant variation in the climate can be attributed due to the large size of the country, its diverse landscapes and also due to The Red Sea coast on the Western side and the Arabian Gulf on the eastern side [3]. A hot whether generally characterizes the Climate of Saudi Arabia for the greater part of the year to northerly winds moving from the eastern Mediterranean towards the Arabian Gulf. Relative humidity is low except along the coastal zones where it reaches over $90 \%$. The average annual temperature is $33.4{ }^{\circ} \mathrm{C}$ in summer and $14{ }^{\circ} \mathrm{C}$ in winter, but there are wide variations. For example, inland temperatures range from below zero at night to a maximum of $50{ }^{\circ} \mathrm{C}$ during summer. In the northern part of Saudi Arabia, temperatures are very high in summer, the hottest month being July, while in south-east regions the hottest month is June and the coldest is JanuaryTable[1]. The vegetation of Saudi Arabia is diverse. Approxematelly2250 species of plant in Saudi Arabia belong to 132 families and 837 genera. About 105 species inhabit dunes, 90 are halophyte, 75 are trees and 12 are aquatic plants. It is also reported that no families or genera in flowering plants are endemic but that are some 246 species that are considered regionally endemic[5].About 450 species of flowering plants in Saudi Arabia have direct benefits to humankind, 45 species out of them are poisonous,334 species are known to have medicinal value,38 species are important palatable fodder plants, Six are important as fuel wood,25 species are human food plants and 47 species are used as ornamentals or for other purposes. Although the Kingdom of Saudi Arabia located within a typical arid to semi-arid region, it is characterized by its unique biological diversity and species that could acclimatize to live under adverse ecological circumstances including weather and dry conditions[6]. Current study area include two physiographic units wadis and sabkah where wadis represent one of the most prominent desert landforms, exhibiting physiographic irregularities that lead to parallel variation in species distribution, Life-form distribution is closely related to topography and landform [7]. Life-form composition is typical of desert flora; the majority of species are therophytes and chamaephytes. Wadi vegetation in general is not constant, it varies from year to year depending upon moisture levels. Generally, growth, regeneration, and distribution of the plant communities in the wadis are controlled by many factors such as geographical position, physiographic features, and human impact [8]. Arabia.

The aim of this research is to studythe distribution of plant species according to soil properties at Wadi Elip that located inSouth Western area of Saudi 
Citation: Ali A. Al- Masaudi, H.S.Al-Zahrani,A.A. Al Toukhy and Hameed Alsamadany, 2018. Plant species distribution and their relation to soil properties in Wadi Elip South Western area in Saudi Arabia. Advances in Environmental Biology., 12(3): 5-9.

Table 1: Mean average of temperature in the study area

\begin{tabular}{|c|c|c|c|c|c|c|c|c|c|c|c|c|}
\hline Months & Jan & Feb & Mar & Apr & May & Jun & Jul & Aug & Sep & Oct & Nov & Des \\
\hline Temperature & 24 & 26 & 29 & 29 & 31 & 32 & 36 & 40 & 35 & 31 & 28 & 27 \\
\hline
\end{tabular}

\section{MATERIALS AND METHODS}

Plant samples andMeasurments:

Twelve stands were selected to represent different soil units (1-7, 8-10 and 11-12 foothill, sand dunes, and sabaka ) respectively in Wadi Elip (Fig 1). The stand size $(20 \times 20) \mathrm{m}$. for each one,list of species, common species were recorded. The abundance and life form were calculated, Braun- Blanquet cover abundance scale as follows:Scale 5, 4, 3, 2, 1, + and r ( rerlay ) cover abundance,75\%, 50-70\%, 25-50\%, 5-25\%, 1-5\%, few and solitary respectively [9, 10].

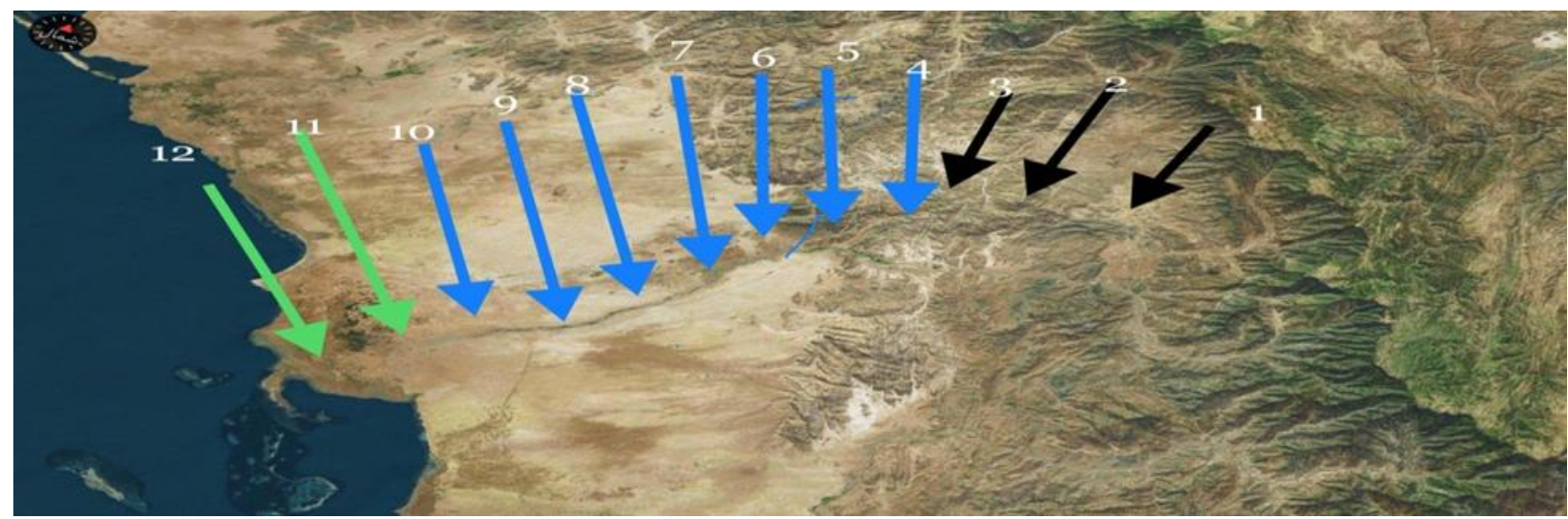

Fig. 1: Distribution of 12 stands along study area by using their coordinates (GPS )

Soil samples and analysis:

In each stand 3 samples of soil were taken at $0-20 \mathrm{~cm}$ soil depth and mixed togeather making representedsoil sample, then itswere air dried and sived by $2 \mathrm{~mm}$ sive. The following analyses: Soil color was identified using Munssel color (1990); Particle size distribution using seving method (USDA, 1991); Chemical analyses which include O.M., ECe, bluk and real density, total porosity and Soluble cations (USDA, 2003); Soil reaction pH (Klut, 1986), were analysiedat the Department of Biological Sciences, Faculty of Science, King Abdulaziz University, Jeddah,Saudi Arabia

Results:

Distribution and Growth Status of Plant Cover:

The oresent study showed thatvegetation at wadi Elip which include three soil unites namely foothill, sand dunes and coastal sabkha where recorded at table (2). The total number of recorded species in all soil unit 40 species belongs to 33 genus and 22 families. Twenty six species representing perennial and Fourteen species were annual with ratios $65 \%$ and $35 \%$ respectively. Fabaceae (leguminosae) have the highest contributions, $17.5 \%$ followed by Boraginaceae and Zygophyllaceaewith ratio (12.50\%) and Poaceae (graminae) with (10\%) and Asteraceae with ratio(5\%)Aizoaceae, Amaranthaceae, Apocynaceae, Asclepiadaceae, Chenopodiaceae, Cucurbitaceae, Cypracea, Malvaceae, Mavaceae, Plumbaginaceae, Polygalaceae, Rhamnaceae, Salvadoraceae, Scrophulariaceae, Solanaceae, Tamaricaceae, Typhaceae, Zygophyllaceae with (2.5\%) for each.Speciesdistrubtion depended on the soil type where Glinuslotoides found only in sand dunes unite, Aervajavanica\&Salvadorapersica were found with high medim low ratios in sand duns, foothill and sabkahresptvally where Zygophyllumcoccineum was found with a high retio in sabkah.

Table.2: Determination the abundance of species according to Braun- Blanquet cover-abundance scalein Wadi Elip

\begin{tabular}{|c|c|c|c|c|c|c|c|c|c|c|c|c|c|c|c|c|c|}
\hline Family & Species & 1 & 2 & 3 & 4 & 5 & 6 & 7 & 8 & 9 & 10 & 11 & 12 & $\mathrm{~T}$ & $\begin{array}{l}\text { Abunda } \\
\text { nce }\end{array}$ & $\begin{array}{l}\text { frequen } \\
\text { cy }\end{array}$ & $\begin{array}{l}\text { densit } \\
\mathrm{y}\end{array}$ \\
\hline Aizoaceae & Glinuslotoides & - & - & - & - & - & 1 & 3 & - & 8 & - & - & - & 12 & 4 & 25 & 1 \\
\hline $\begin{array}{l}\text { Amarantha } \\
\text { ceae }\end{array}$ & Aervajavanica & 9 & - & 3 & 9 & 17 & 2 & 8 & 13 & 12 & 3 & 8 & - & 84 & 8.4 & 83.30 & 5.3 \\
\hline $\begin{array}{l}\text { Apocynace } \\
\text { ae }\end{array}$ & Calotropisprocera & 3 & 8 & 0 & 2 & 9 & 2 & 3 & 4 & 5 & 7 & 0 & 1 & 44 & 3.6 & 83.30 & 3.6 \\
\hline $\begin{array}{l}\text { Asclepiada } \\
\text { ceae }\end{array}$ & $\begin{array}{l}\text { Leptadeniapyrotec } \\
\text { hnica }\end{array}$ & - & 5 & 4 & - & 4 & - & 6 & - & 2 & - & 3 & - & 33 & 5.5 & 83.30 & 3.5 \\
\hline \multirow[t]{2}{*}{ Asteraceae } & $\begin{array}{l}\text { Rhanteriumepappo } \\
\text { sum }\end{array}$ & 6 & 4 & 2 & 3 & 9 & 6 & 13 & 8 & 10 & 0 & 7 & 8 & 76 & 6.9 & 91.60 & 6.3 \\
\hline & palicania vulgaris & - & 2 & - & - & - & & 5 & 3 & 3 & - & - & - & 13 & 3.25 & 33.3 & 1.08 \\
\hline \multirow[t]{5}{*}{$\begin{array}{l}\text { Boraginace } \\
\text { ae }\end{array}$} & $\begin{array}{l}\text { Heliotropiumeurop } \\
\text { aeum }\end{array}$ & 5 & 9 & 7 & - & 3 & - & - & 5 & - & 4 & 7 & - & 40 & 5.7 & 58.3 & 3.3 \\
\hline & $\begin{array}{l}\text { Heliotropiumcrisp } \\
\text { um }\end{array}$ & -- & - & - & - & - & 4 & - & 6 & 5 & - & 2 & 3 & 20 & 4 & 41.66 & 2 \\
\hline & $\begin{array}{l}\text { Trichodesmaehren } \\
\text { bergii }\end{array}$ & - & - & 3 & 8 & 6 & 2 & 5 & 8 & - & - & - & - & 32 & 5.3 & 50 & 2.6 \\
\hline & $\begin{array}{l}\text { Heliotropiumcuras } \\
\text { savicum }\end{array}$ & 2 & 6 & 5 & 5 & 7 & 5 & 10 & 5 & - & 5 & - & - & 50 & 5.50 & 75 & 5.6 \\
\hline & $\begin{array}{l}\text { Heliotropiumlongif } \\
\text { lorum }\end{array}$ & 4 & 4 & 6 & 9 & - & - & 7 & 4 & 8 & 6 & 7 & 4 & 58 & 5,8 & 83.3 & 7 \\
\hline $\begin{array}{l}\text { Chenopodia } \\
\text { ceae }\end{array}$ & Suaedafruticosa & 8 & - & 2 & - & 4 & 5 & 3 & 6 & - & 5 & 8 & 4 & 45 & 5 & 75 & 3.75 \\
\hline $\begin{array}{l}\text { Cucurbitace } \\
\text { ae }\end{array}$ & $\begin{array}{l}\text { citrulluscolocynthi } \\
\text { s }\end{array}$ & 2 & 3 & - & - & 7 & 9 & 4 & - & 12 & 4 & 7 & - & 48 & 6 & 66.6 & 4 \\
\hline Cypracea & $\begin{array}{l}\text { Heliotropiumlongif } \\
\text { lorum }\end{array}$ & 4 & - & 4 & 5 & 2 & 7 & - & 11 & 6 & 3 & 7 & '9 & 58 & 5.8 & 83.3 & 4.8 \\
\hline
\end{tabular}


Citation: Ali A. Al- Masaudi, H.S.Al-Zahrani,A.A. Al Toukhy and Hameed Alsamadany, 2018. Plant species distribution and their relation to soil properties in Wadi Elip South Western area in Saudi Arabia. Advances in Environmental Biology., 12(3): 5-9.

\begin{tabular}{|c|c|c|c|c|c|c|c|c|c|c|c|c|c|c|c|c|c|}
\hline \multirow[t]{7}{*}{ Fabaceae } & $\begin{array}{l}\text { Acacia } \\
\text { ehrenbergiana }\end{array}$ & 5 & 9 & 7 & 3 & - & - & 9 & 3 & 9 & 5 & 2 & - & 50 & 5.50 & 75 & 6.08 \\
\hline & Senna alexandrina & - & 7 & 6 & - & 5 & 7 & 8 & 6 & 7 & 4 & 7 & - & 57 & 6.3 & 75 & 6.25 \\
\hline & Acacia italic & 3 & 1 & 2 & 2 & - & - & 2 & 3 & 7 & - & - & - & 19 & 2.7 & 58 & 1.5 \\
\hline & Tephrosianubica & - & - & 9 & - & 4 & 6 & - & - & 8 & 7 & 5 & 4 & 44 & 6.2 & 58 & 3.6 \\
\hline & Sesbaniasesban & - & - & - & - & - & - & - & 3 & 8 & 6 & 10 & 9 & 36 & 7.2 & 41 & 3 \\
\hline & Prosopis juliflora & 3 & 8 & 11 & 9 & 7 & 11 & 8 & 6 & 8 & 4 & 5 & - & 80 & 7.2 & 91.6 & 6.6 \\
\hline & Juncuspunctorius & 6 & - & 4 & 5 & 9 & - & 7 & 8 & - & 7 & 5 & 2 & 53 & 5.8 & 75 & 4.4 \\
\hline Malvaceae & Datura innoxia & - & - & 7 & 3 & 8 & 2 & 2 & 7 & 3 & - & - & - & 32 & 4.5 & 50 & 3.8 \\
\hline Mavaceae & Abutilon muticum & 4 & 3 & - & - & 6 & 13 & 5 & 10 & 3 & 4 & 8 & - & 56 & 6.2 & 75 & 4.6 \\
\hline $\begin{array}{l}\text { Plumbagina } \\
\text { ceae }\end{array}$ & $\begin{array}{l}\text { Limoniumcylindrif } \\
\text { olium }\end{array}$ & - & - & - & - & 7 & - & - & - & 8 & 2 & 4 & 5 & 26 & 5.2 & 41 & 2.1 \\
\hline \multirow[t]{4}{*}{ Poacea } & Cenchrusciliaris & 1 & - & - & 11 & 5 & 10 & 12 & 5 & 7 & 5 & - & 3 & 59 & 6.5 & 58 & 4.8 \\
\hline & Panicum turgidum & 7 & 3 & - & - & - & - & & 6 & 2 & 2 & - & - & 20 & 4 & 41 & 1.6 \\
\hline & Chlansgayana & 1 & 3 & 12 & 6 & - & - & 10 & 7 & 12 & 9 & - & 6 & 66 & 7.3 & 75 & 5.5 \\
\hline & $\begin{array}{l}\text { Aristidaadscension } \\
\text { is }\end{array}$ & 9 & 7 & - & 7 & 3 & 8 & 8 & 3 & - & 9 & 5 & - & 59 & 6.50 & 75 & 6.08 \\
\hline $\begin{array}{l}\text { Polygalacea } \\
\mathrm{e}\end{array}$ & Polygala erioptera & - & - & - & 10 & 6 & 12 & 6 & 2 & 2 & - & - & - & 38 & 6.3 & 50 & 3.2 \\
\hline $\begin{array}{l}\text { Rhamnacea } \\
\text { e }\end{array}$ & $\begin{array}{l}\text { Ziziphus spina- } \\
\text { christi }\end{array}$ & - & - & 2 & - & - & 4 & 6 & - & 5 & 3 & - & - & 20 & 4 & 41 & 1.6 \\
\hline $\begin{array}{l}\text { Salvadorac } \\
\text { eae }\end{array}$ & Salvadorapersica & - & 10 & 9 & 8 & 13 & 8 & 9 & 13 & 10 & 11 & 10 & - & 71 & 7.1 & 83 & 5.9 \\
\hline $\begin{array}{l}\text { Scrophulari } \\
\text { aceae }\end{array}$ & Scopariadulcis & 2 & 4 & 4 & - & 14 & 19 & 5 & 12 & 8 & - & 9 & 4 & 81 & 8.1 & 83 & 6.75 \\
\hline Solanaceae & Solanum nigrum & - & - & - & 3 & 4 & 7 & 5 & 2 & 8 & - & - & - & 29 & 4.8 & 50 & 2.4 \\
\hline $\begin{array}{l}\text { Tamaricace } \\
\text { ae }\end{array}$ & Tamarixnilotica & - & - & 8 & 7 & 6 & 11 & 3 & 4 & 9 & 7 & - & - & 55 & 6.8 & 66 & 4.5 \\
\hline Typhaceae & Typhadomingensis & - & - & - & 7 & 13 & 9 & 6 & 9 & 3 & - & - & -- & 47 & 7.8 & 50 & 2 \\
\hline \multirow[t]{5}{*}{$\begin{array}{l}\text { Zygophylla } \\
\text { ceae }\end{array}$} & $\begin{array}{l}\text { Zygophyllum } \\
\text { simplex }\end{array}$ & 6 & - & - & - & 3 & 4 & - & - & 3 & - & - & - & 16 & 4 & 33.3 & 1.3 \\
\hline & $\begin{array}{l}\text { Zygophyllumcocci } \\
\text { neum }\end{array}$ & 2 & - & - & - & 4 & 9 & 3 & 12 & - & 9 & 4 & 10 & 53 & 6.6 & 66 & 4.4 \\
\hline & $\begin{array}{l}\text { Indigofera doll } \\
\text { longifolia }\end{array}$ & - & - & - & 3 & 7 & - & 9 & 8 & 7 & - & - & - & 34 & 6.8 & 41 & 2.8 \\
\hline & Faganiaindica & - & - & - & - & 17 & 12 & 12 & 9 & - & - & - & - & 31 & 7.7 & 33.3 & 2.58 \\
\hline & Peganum harmala & 8 & 9 & 10 & 9 & 6 & 5 & 2 & 11 & 8 & - & - & - & 68 & 7.5 & 75 & 5.66 \\
\hline
\end{tabular}

Soil morphological characteristics:

Soil morphological characteristics are shown in Table (3). The soil types in the study area were determined to be immature sand,sand loam and silty loam.Their horizon sequences were described as layer at a depth from 0 to $20 \mathrm{~cm}$ the surface layers were formed mainly of coarse sand and silty in all study areas and were covered by herbs as plant cover species..Soil texture class varied from sandy, loamy-sandy to silty sand. Bulk density ranged from 1.38 to $1.75 \mathrm{~g} / \mathrm{cm} 3$ while real density. varied from 2.51 to $2.68 \mathrm{~g} / \mathrm{cm} 3$. Total porosity ranging between 34.70 and $45.01 \%$.

Table 3: Some morphological and physical characteristics of the studied soil in Wadi Elip

\begin{tabular}{|c|c|c|c|c|c|c|c|c|c|c|c|}
\hline \multirow[b]{2}{*}{ St. } & \multirow{2}{*}{ Depth } & \multicolumn{2}{|l|}{ Colure } & \multirow{2}{*}{ Texture } & \multicolumn{3}{|c|}{ consistence } & \multirow{2}{*}{$\begin{array}{l}\text { Structur } \\
\mathrm{e}\end{array}$} & \multirow{2}{*}{$\begin{array}{l}\text { R.D } \\
\mathrm{g} / \mathrm{cm}^{3}\end{array}$} & \multirow{2}{*}{$\begin{array}{l}\text { B.D } \\
\mathrm{g} / \mathrm{cm}^{3}\end{array}$} & \multirow{2}{*}{$\begin{array}{l}\text { Total } \\
\text { prosity } \%\end{array}$} \\
\hline & & Dry & Moist & & soft & Stiky & plastic & & & & \\
\hline 1 & $1-20$ & $\begin{array}{l}\text { 10YR514 Light } \\
\text { yellowish brown }\end{array}$ & $\begin{array}{l}\text { 10YR514 Light } \\
\text { yellowish brown }\end{array}$ & Sand & loose & $\begin{array}{l}\text { Non } \\
\text { sticky }\end{array}$ & $\begin{array}{l}\text { Non } \\
\text { plastic }\end{array}$ & $\begin{array}{l}\text { Slinght } \\
\text { green }\end{array}$ & 2.80 & 1.12 & 60.00 \\
\hline 2 & $1-20$ & $\begin{array}{l}\text { 10YR516 Light } \\
\text { yellowish brown }\end{array}$ & $\begin{array}{l}\text { 10YR316 dark } \\
\text { yellowish brown }\end{array}$ & $\begin{array}{l}\text { Sand } \\
\text { loamy }\end{array}$ & soft & $\begin{array}{l}\text { Slightly } \\
\text { sticky }\end{array}$ & $\begin{array}{l}\text { Slightly } \\
\text { plastic }\end{array}$ & $\begin{array}{l}\text { Slinght } \\
\text { green }\end{array}$ & 2.28 & 1.07 & 53.07 \\
\hline 3 & $1-20$ & $\begin{array}{l}\text { 10YR516 Light } \\
\text { yellowish brown }\end{array}$ & $\begin{array}{l}\text { 10YR316 dark } \\
\text { yellowish brown }\end{array}$ & $\begin{array}{l}\text { Sand } \\
\text { loamy }\end{array}$ & soft & $\begin{array}{l}\text { slightly } \\
\text { sticky }\end{array}$ & $\begin{array}{l}\text { Slightly } \\
\text { plastic }\end{array}$ & $\begin{array}{l}\text { Singht } \\
\text { green }\end{array}$ & 2.59 . & .1 .17 & 54 \\
\hline 4 & $1-20$ & $\begin{array}{l}\text { 10YR514 Light } \\
\text { yellowish brown }\end{array}$ & $\begin{array}{l}\text { 10YR514 Light } \\
\text { yellowish brown }\end{array}$ & Sand & loose & Non stiky & $\begin{array}{l}\text { Non } \\
\text { plastic }\end{array}$ & $\begin{array}{l}\text { Slinght } \\
\text { green }\end{array}$ & 2.65 & 1.11 & 58 \\
\hline 5 & $1-20$ & $\begin{array}{l}\text { 10YR516 Light } \\
\text { yellowish brown }\end{array}$ & $\begin{array}{l}\text { 10YR316 dark } \\
\text { yellowish brown }\end{array}$ & $\begin{array}{l}\text { Sand } \\
\text { loamy }\end{array}$ & loose & $\begin{array}{l}\text { Slight } \\
\text { sticky }\end{array}$ & $\begin{array}{l}\text { Slightly } \\
\text { plastic }\end{array}$ & $\begin{array}{l}\text { Slinght } \\
\text { green }\end{array}$ & 2.78 & 1.33 & 52 \\
\hline 6 & $1-20$ & $\begin{array}{l}\text { 10YR514 Light } \\
\text { yellowish brown }\end{array}$ & $\begin{array}{l}\text { 10YR514 Light } \\
\text { yellowish brown }\end{array}$ & Sand & loose & Non stiky & $\begin{array}{l}\text { Non } \\
\text { plastic }\end{array}$ & $\begin{array}{l}\text { Slinght } \\
\text { green }\end{array}$ & 2.88 & 1.07 & 62.85 \\
\hline 7 & $1-20$ & $\begin{array}{l}\text { 10YR516 Light } \\
\text { yellowish brown }\end{array}$ & $\begin{array}{l}\text { 10YR316 dark } \\
\text { yellowish brown }\end{array}$ & $\begin{array}{l}\text { Sand } \\
\text { loamy }\end{array}$ & $\begin{array}{l}\text { Loos } \\
\mathrm{e}\end{array}$ & $\begin{array}{l}\text { Slightly } \\
\text { sticky }\end{array}$ & $\begin{array}{l}\text { Slightly } \\
\text { plastic }\end{array}$ & $\begin{array}{l}\text { Slinght } \\
\text { green }\end{array}$ & 2.33 & 1.10 & 52.79 \\
\hline 8 & $1-20$ & $\begin{array}{l}\text { 10YR516 Light } \\
\text { yellowish brown }\end{array}$ & $\begin{array}{l}\text { 10YR316 dark } \\
\text { yellowish brown }\end{array}$ & $\begin{array}{l}\text { Sand } \\
\text { loamy }\end{array}$ & loose & $\begin{array}{l}\text { slightly } \\
\text { sticky }\end{array}$ & $\begin{array}{l}\text { Slightly } \\
\text { plastic }\end{array}$ & $\begin{array}{l}\text { Slinght } \\
\text { green }\end{array}$ & 2.60 & 1.30 & 50 \\
\hline 9 & $1-20$ & $\begin{array}{l}\text { 10YR516 Light } \\
\text { yellowish brown }\end{array}$ & $\begin{array}{l}\text { 10YR316 dark } \\
\text { yellowish brown }\end{array}$ & $\begin{array}{l}\text { Sand } \\
\text { loamy }\end{array}$ & loose & $\begin{array}{l}\text { slightly } \\
\text { sticky }\end{array}$ & $\begin{array}{l}\text { Slightly } \\
\text { plastic }\end{array}$ & $\begin{array}{l}\text { Slinght } \\
\text { green }\end{array}$ & 2.54 & 1.21 & 52 \\
\hline 10 & $1-20$ & $\begin{array}{l}\text { 10YR516 Light } \\
\text { yellowish brown }\end{array}$ & $\begin{array}{l}\text { 10YR316 dark } \\
\text { yellowish brown }\end{array}$ & $\begin{array}{l}\text { Sand } \\
\text { loamy }\end{array}$ & loose & $\begin{array}{l}\text { slightly } \\
\text { sticky }\end{array}$ & $\begin{array}{l}\text { Slightly } \\
\text { plastic }\end{array}$ & $\begin{array}{l}\text { Slinght } \\
\text { green }\end{array}$ & 2.55 & 1.25 & 50 \\
\hline 11 & $1-20$ & $\begin{array}{l}\text { 10YR316 dark } \\
\text { yellowish brown }\end{array}$ & $\begin{array}{l}\text { 10YR313 dark } \\
\text { brown }\end{array}$ & $\begin{array}{l}\text { Silt } \\
\text { loamy }\end{array}$ & soft & $\begin{array}{l}\text { moderatel } \\
\text { y sticky }\end{array}$ & $\begin{array}{l}\text { Slightly } \\
\text { plastic }\end{array}$ & massive & 2.45 & 1.40 & 42.86 \\
\hline 12 & $1-20$ & $\begin{array}{l}\text { 10YR316 dark } \\
\text { yellowish brown }\end{array}$ & $\begin{array}{l}\text { 10YR313 dark } \\
\text { brown }\end{array}$ & $\begin{array}{l}\text { Silt } \\
\text { loamy }\end{array}$ & soft & $\begin{array}{l}\text { moderatel } \\
\text { y sticky }\end{array}$ & $\begin{array}{l}\text { Slightly } \\
\text { plastic }\end{array}$ & massive & 2.40 & 1.35 & 43.75 \\
\hline
\end{tabular}


Citation: Ali A. Al- Masaudi, H.S.Al-Zahrani,A.A. Al Toukhy and Hameed Alsamadany, 2018. Plant species distribution and their relation to soil properties in Wadi Elip South Western area in Saudi Arabia. Advances in Environmental Biology., 12(3): 5-9.

The soil chemical propertiesare shown in Table (4). The soil reaction $(\mathrm{pH})$ of soil in the study areas displayed in a relatively normal range $(6.7,7.20$ and 8.40); however, the $\mathrm{pH}$ of the soil analyses indicated that it is moderately basic. Electreicalcondvity were higher in the coastal sabkha $(8.20 \mathrm{Desm} . / \mathrm{m})$ and very low in foothill $(0.10 \mathrm{Desm} . \mathrm{m})$, it is low to modertily in sand duans $(0.9-3.4 \mathrm{Desm} . \mathrm{m})$ Table(4).The contents of organic carbone was highly in Coastal sabkha near red sea $(2.60 \%)$ and slightly highly in foothill and coastal sand dunes $(1.98 \%)$ and $(1.73 \%)$ respectfillyTable (4).The concentrations of cations in the surface layer (0-20) were generally low in foothill and coastal sand dunes but it increased at coastal sabkha.Concentrations of $\mathrm{Ca}, \mathrm{Mg}, \mathrm{Na}, \mathrm{K}$ and $\mathrm{p}$ were recorded in table (4) rsults showed that all element concentrations were higher in the coastal sabkha more than foothill and coastal sand dunes in Wadi Elip.The concentrations of $\mathrm{Ca}^{2+}$ in the coastal sabkha ranging from 0.81 to $1.05 \mathrm{mg} / \mathrm{gm}$, it was ranging from 0.02 to $0.04 \mathrm{meg} / \mathrm{g}$ in foothill \&costal sand dunes,Magnesium had the concentrations ranged between 0.02 to $1.40 \mathrm{mg} / \mathrm{gm}$ in coastal sabkha, $0.02-0.04 \mathrm{mg} / \mathrm{g}$ in foothill and coastal sand dunes. However, the $\mathrm{Na}^{+}$concentrations were the highest among the four cation concentrations in coastal sabkha, where the concentration ranged from $3.39-9.34 \mathrm{mg} / \mathrm{g}$. The concentrations of $\mathrm{K}^{+}$ranged from $0.10-$ $1.00 \mathrm{mg} / \mathrm{g}$ in coastal sabkha where it ranged from 0.01 to $0.11 \mathrm{mg} / \mathrm{gm}$. in foothill and coastal sand dunes. The concentration of $\mathrm{p}(3.04 \mathrm{mg} / \mathrm{g})$ it was a higher in coastal sabkha than other two units or foothill and coastal sand dunes where it ranged between 0.08 to $1.75 \mathrm{mg} / \mathrm{g}$. $\mathrm{Zn}$ ranged from 0.17 to $0.70 \mathrm{mg} / \mathrm{gm}$ at foothill and coastal sand dunes where it ranged from 0.05 to $0.50 \mathrm{meg} / \mathrm{g}$ in coastal sabkha.

Table 4: Mechanial analyses of soil horizons and moisture content under different plant communities at Wadi Elip

\begin{tabular}{|c|c|c|c|c|c|c|c|c|c|c|c|c|c|c|c|}
\hline \multirow{2}{*}{ Sta. } & \multirow{2}{*}{$\begin{array}{l}\text { Dep } \\
\mathrm{cm})\end{array}$} & \multirow{2}{*}{ Ec } & \multirow{2}{*}{$\mathrm{pH}$} & \multirow{2}{*}{$\begin{array}{l}\text { organic matter } \\
\%\end{array}$} & \multicolumn{5}{|c|}{ Menaral Cations (mg / gm) } & \multicolumn{6}{|c|}{ Heavy mattleCations (mg / gm) } \\
\hline & & & & & $\mathrm{Mg}$ & $\mathrm{Ca}$ & $\mathrm{Na}$ & $\mathrm{K}$ & $\mathrm{P}$ & $\mathrm{Ba}$ & $\mathrm{Zn}$ & $\mathrm{Mn}$ & $\mathrm{Cu}$ & $\mathrm{Fa}$ & $\mathrm{Ni}$ \\
\hline 1 & $1-20$ & 0.10 & $\begin{array}{l}6.7 \\
0\end{array}$ & 1.60 & 0.018 & 0.083 & 0.300 & 0.005 & 0.075 & 0.025 & 0.650 & 0.001 & 0.095 & 0.005 & 0.035 \\
\hline 2 & $1-20$ & 0.10 & $\begin{array}{l}6.7 \\
0 \\
\end{array}$ & 1.80 & 0.018 & 0.101 & 0.274 & 0.070 & 0.390 & 0.060 & 0.320 & 0.001 & 0.165 & 0.045 & 0.040 \\
\hline 3 & $1-20$ & 0.10 & $\begin{array}{l}6.8 \\
0\end{array}$ & 1.90 & 0.027 & 0.129 & 0.213 & 0.103 & 1.750 & 0.085 & 0.365 & 0.001 & 0.200 & 0.065 & 0.005 \\
\hline 4 & $1-20$ & 0.20 & $\begin{array}{l}6.8 \\
0\end{array}$ & 1.60 & 0.023 & 0.125 & 0.321 & 0.214 & 1.465 & 0.100 & 0.355 & 0.001 & 0.265 & 0.050 & 0.040 \\
\hline 5 & $1-20$ & 0.90 & $\begin{array}{l}7.1 \\
0 \\
\end{array}$ & 1.90 & 0.025 & 0.088 & 0.328 & 0.166 & 0.075 & 0.120 & 0.015 & 0.001 & 0.280 & 0.240 & 0.075 \\
\hline 6 & $1-20$ & 0.80 & $\begin{array}{l}7.1 \\
0\end{array}$ & 2.50 & 0.035 & 0.204 & 0.434 & 0.156 & 0.080 & 0.235 & 0.745 & 0.025 & 0.075 & 0.080 & 0.055 \\
\hline 7 & $1-20$ & 2.10 & $\begin{array}{l}7.2 \\
0\end{array}$ & 1.50 & 0.019 & 0.108 & 0.292 & 0.085 & 0.175 & 0.085 & 0.309 & 0.140 & 0.130 & 0.315 & 0.080 \\
\hline 8 & $1-20$ & 1.80 & $\begin{array}{l}7.3 \\
0\end{array}$ & 1.70 & 0.023 & 0.099 & 0.359 & 0.101 & 0.015 & 0.085 & 0.510 & 0.025 & 0.190 & 0.040 & 0.065 \\
\hline 9 & $1-20$ & $2 . .20$ & $\begin{array}{l}7.3 \\
0\end{array}$ & 2.30 & 0.016 & 0.099 & 0.227 & 0.089 & 0.225 & 0.075 & 0.585 & 0.110 & 0.140 & 1.165 & 0.050 \\
\hline 10 & $1-20$ & 3.40 & $\begin{array}{l}7.5 \\
0\end{array}$ & 2.05 & 0.019 & 0.136 & 0.168 & 0.105 & 1.175 & 0.080 & 0.165 & 0.001 & 0.125 & 0.001 & 0.035 \\
\hline 11 & $1-20$ & 5.60 & $\begin{array}{l}7.9 \\
0\end{array}$ & 2.30 & 0.016 & 0.081 & 3939 & 0.104 & 0.010 & 0.080 & 0.485 & 0.001 & 0.110 & 0.430 & 0.040 \\
\hline 12 & $1-20$ & 8.20 & $\begin{array}{l}8.4 \\
0\end{array}$ & 2.60 & 1.372 & 1.049 & 9.335 & 0.975 & 3.040 & 0.745 & 0.050 & 0.100 & 0.065 & 0.001 & 0.00 \\
\hline
\end{tabular}

Discussion:

The plant communities of the surveyed transect can be classified into three types for each unit study area, that was, Aervajavanica, and Prosopis juliflorain foothills area,Salvadorapersicafound coastal sand dunes (meidle study area ), Suaedafruticosain coastal sabkha( near The Red Sea ). A total of 40 plant species belonging to 23 family were collected and identified from the study area. There are significant differences in the richness of vegetation between the different soil units. This could be attributed to the influence of altitude, humidity or the highest levels of soil nutrients [14]. Chemical analysis revealed that, the coastal sabkha( near The Red Sea) locations characterized by having high amount of organic matter, this may be due to the mineral nature of soil deposits. Additionally, it is noteworthy that different studies recorded that available contents of $\mathrm{N}, \mathrm{P}, \mathrm{K}$. Fe, Mn, in general, lie at a very high level [15-20].Vegetationon the foothill and coastal sand dunes were characterized as coarse to fine sands in soil samples (Table 4$)$. The values of the six cations in foothill area were $(1.75 \mathrm{mg} / \mathrm{g}) \mathrm{P}^{3+}>(0.33$ $\mathrm{mg} / \mathrm{g}) \mathrm{Na}^{+}>(0.21 \mathrm{mg} / \mathrm{g}) \mathrm{K}^{+}>(0.13 \mathrm{mg} / \mathrm{g}) \mathrm{Ca}^{2+}>(0.03) \mathrm{Mg}^{2+>}(0.005 \mathrm{mg} / \mathrm{g}) \mathrm{Fe}^{2+}$, where in costal sand duens values were $(1.17 \mathrm{mg} / \mathrm{g}) \mathrm{Fe}^{2+}>(0.43 \mathrm{mg} / \mathrm{g}) \mathrm{Na}{ }^{+}>(0.23$ $\mathrm{mg} / \mathrm{g}) \mathrm{P}^{3+}>(0.11 \mathrm{gm} / \mathrm{g}) \mathrm{K}^{+}(0.04 \mathrm{mg} / \mathrm{g}) \mathrm{Mg}^{2+}>(0.02 \mathrm{mg} / \mathrm{g}) \mathrm{Ca}^{2+}$, finally incostal Sabkha $\mathrm{Na}^{2+}>\mathrm{P}^{3+}>\mathrm{Mg}^{2+}>\mathrm{Ca}^{2+}>\mathrm{K}^{+}>\mathrm{Fe}^{2+}$ it is values were $9.34,3.04,1.37,1.05$ and $0.98 \mathrm{mg} / \mathrm{g}$ respectivelly (Table 2 ). The highP ${ }^{3+} \mathrm{Fe}^{2+}$ and $\mathrm{Na}^{+}$concentrations in soil might be because the plant covers preferentially limit the absorption of available $\mathrm{P}^{3+} . \mathrm{Fe}^{2+}$ and $\mathrm{Na}^{+}$from the soil [8] and the absorbed might be incorporated into plant covers biomass and only a smallP ${ }^{3+}$.Fe ${ }^{2+}$ and $\mathrm{Na}^{+}$quantity being resupplied to the plant cover floor through litter-fall. The deep groundwater level and temperature in this area might be interfering with litter decomposition. Meanwhile, the concentrations of $\mathrm{Mg}^{2+}$ in this study area especially costal sabkha might be because of the contribution from sea salt. Also Na ${ }^{+}$concentration was the higest one, Na+ showed the highest saturation under Suaedafruticosaplant. Some species share the presence in the two locations: Aervajavanica, and Prosopis juliflora in foothills area, Salvadorapersica and Aervajavanica found coastal sand dunes (meidle study area ), Suaedafruticosa and Prosopis juliflora in coastal sabkha( near The Red Sea). This attributed to the presence of the same chemical constituents found in the two locations. There is a clear correlation between organic matter and the distribution of different plant species. Deficiency of one or more element in the soil is one of the major reasons for poor cultivation of soils[20 -29]. The floristic and chemical composition of soil data outlined above clearly indicate that Wadi Elip is rich, with variable vegetation.

\section{Conclusion:}

Wadi Elip include three soil unites namely foothill, sand dunes and coastal sabkha, 40 species belongs to 33 genus and 22 families were recorded. Twenty six species representing perennial and Fourteen species were annual with ratios $65 \%$ and $35 \%$ respectively. Species distrubtion depended on soil type where Glinuslotoides found only in foothill unite, Aervajavanica\&Salvadorapersica in sand dunsand Zygophyllumcoccineum in sabkahrespectivelly. The results of the present work strongly suggested or enhanced more investigations on the ecology of this widely distributed species. This work will be followed by a future study on mapping the plant communities along the same area to make a fulldescription of their structure, distribution and location of each community in relation to the chemical constituents ofthe soil.

\section{REFERENCES}

[1] Al-Sherif, E.A., A.M. Ayesh and S.M. Rawi, 2013. Floristic composition, life form and chorology ofplant life at Khulais region, western Saudi Arabia, Pak. J. Bot., 45(1): 29-38.

[2] Mohamed Moawed1,Abid Ansari1, Wild plants diversity of Red Sea coastal region Tabuk of the Saudi Arabia, University of Tabuk, Tabuk, Saudi Arabia, 2015

[3] Thomas, A., 2011. Prelude to the plant diversity of Saudi Arabia plantdiversityofsaudiarabia.info/ Biodiversity-Saudi- GMBH., pp: 130. 
Citation: Ali A. Al- Masaudi, H.S.Al-Zahrani,A.A. Al Toukhy and Hameed Alsamadany, 2018. Plant species distribution and their relation to soil properties in Wadi Elip South Western area in Saudi Arabia. Advances in Environmental Biology., 12(3): 5-9.

[4] Abd El-Raham, A.A., 1986. The desert of the Arabian Peninsula, hot desert and arid shrubland.Ecosystem of the World, 12 (B): 29 B 55.

[5] Al-Ghamdi; A Bawadekji, Saudi J Biol. Sci., 2014, 21: 554-565.

[6] Al-Sherif, E.A., A.M. Ayesh and S.M. Rawi, 2014. Floristic composition, life form and chorology of plant life at Khulais region, western Saudi Arabia. Pak. J. Bot., 45(1): 29-38.

[7] Chaudhary SA. In: Flora of the Saudi Arabia Kingdom. Ministry of Agriculture and Water press, Riyadh. 2001;1-3.

[8] Fakhireh, A., M. Ajorlo, A. Shahryari, S. Mansouri, S. Nouri, A. Pahlavanravi, 2012. The autecological characteristics of Desmostachyabipinnata in hyperarid regions. Turk J Botany, 36: 690-696.

[9] Korkmaz M and H. Özçelik, 2011a. Systematical and morphological characteristics of annual Gypsophila L. (Caryophyllaceae) taxa of Turkey. Biological Diversity and Conservation (Biodicon) 4: 79-9.

[10] Mueller-Dombois, D. and H. Ellenberg, 1974. Aims and Methods of Vegetation Ecology. John Wiley and Sons, New York, pp: 547.

[11] USDA, 2010. Keys to soil Taxonomy. 11th Edition United States Department of Agriculture, (USDA),USA.

[12] USDA, 2003. Soil Survey Manual. United States Departments of Agriculture (USAD), Handbook 18,V.S..Print. of Washington, DC., USA

[13] Wang ZY, Qiu QQ, Seufert W, Taguchi T, Testa JR, Whitmore SA, Callen DF, Welsh D, Shenk T, Deuel TF. 1996. Molecular cloning of the cDNA and chromosome localization of the gene for human ubiquitin-conjugating enzyme. Journal of Biological Chemistry. Oct 4;271(40):24811-6.

[14] Fayed, A., K. Zayed, 1989. Vegetation alongMakkah-Taif road, Saudi Arabia. Arab Gulf J. Sci.Res., 7: 97-117.

[15] Jackson, M.L., 1962. Soil chemical analysis. Constable and Co. Ltd. London, pp: 67.

[16] Karpoff, R., 1957. Soil B geology of Arabian region. Bull. Soc. Geol. France., 6: 653-697.

[17] El-Bana, M.I., A. Al-Mathnani, 2009. Vegetation-soil relationships in the Wadi Al-Hayat area of the LibyanSahara. Australian Journal of Basic and Applied Sciences, 3: 740-747.

[18] Al-Zahrani, S.A., 2011. Effect of pouring sewage water in the red sea coast on the accumulation ofheavy metals in coastal plants. MSc thesis, Department of Biological Sciences, Faculty ofScience, King Abdulaziz University, Saudi Arabia.

[19] Munsell Soil Color Chants, 1990. Determination of Soil Color. U.S Dept. Agric. Handbook 18.

[20] Robert, W.D., 2008. Soil Testing Manual Procedures, Classification Data and Sampling practices.

[21] Printed by press of Ohio. Rossiter DG, Van Wambeke AR 1997. Automate land evaluation system (ALES). Version 4.65 user's manual Cornell Univ., Soil Dept., Crop. Atmosphere Science "SCAS". Teaching series no T 93-2 revision 6 Ithaca NYVSA.

[22] Smith, G., 1986. The Guy Smith interviews: Rational for concepts in soil taxonomy USDA. S.M.S.S,Tech. Monograph No11,Washington, DC, USA.

[23] Sys, C., W. Verheye, 1978. An attempt to the evaluation of physical and characteristics for irrigationaccording to the FAO Framework for land evaluation. Int. Train Ceut. For Postgraduate, SoilScientists.

[24] Sys, C., E. Van Rant, J. Debavey, 1991. Land Evaluation part I and II Lecture notes. GheutUniv.,Ghent Belgium.

[25] ZAR EL Karemy, K.M. Zayed, 1995. Distribution of Plant Communities Across AL Baha, SW Saudi Arabia.

[26] Laurent, D., 1993. Kingdom of Saudi Arabia Atlas ofIndustrial minerals. Saudi Arabia, Ministry ofPetroleum and Mineral Resources, DirectorateGeneral of mineral Resources, Jeddah, Saudi Arabiapp, 7-10 22.

[27] Mohamed, A. And M. El - Tom, 1985. Ecologicalrelationships of some vegetation units in theJeddah-Makkah region, Saudi Arabia. Arab Gulf J.Sci. Res., 3: $607-622$. 